(1)

\title{
IL-33-driven ILC2/eosinophil axis in fat is induced by sympathetic tone and suppressed by obesity
}

\author{
Xiaofeng Ding1,2, Yan Luo1,3, Xing Zhang1,4, Handong Zheng ${ }^{4}$, Xin Yang', \\ Xuexian Yang 4 and Meilian Liu ${ }^{1,3}$ \\ 'Department of Biochemistry and Molecular Biology, University of New Mexico Health Sciences Center, \\ Albuquerque, New Mexico, USA \\ ${ }^{2}$ Key Laboratory of Protein Chemistry and Development Biology of State Education Ministry of China, \\ College of Life Science, Hunan Normal University, Changsha, Hunan, China \\ ${ }^{3}$ Department of Metabolism and Endocrinology, Metabolic Syndrome Research Center, Key Laboratory \\ of Diabetes Immunology, Ministry of Education, National Clinical Research Center for Metabolic \\ Diseases, The Second Xiangya Hospital, Central South University, Changsha, Hunan, China \\ ${ }^{4}$ Department of Molecular Genetics and Microbiology, University of New Mexico Health Sciences Center, \\ Albuquerque, New Mexico, USA
}

Correspondence should be addressed to $\mathrm{X}$ Yang or M Liu Email xyang@salud.unm.edu or meilianliu@salud.unm.edu

\begin{abstract}
Group 2 innate lymphoid cells (ILC2s) in white adipose tissue (WAT) promote WAT browning and assist in preventing the development of obesity. However, how ILC2 in adipose tissue is regulated remains largely unknown. Here, our study shows that ILC2s are present in brown adipose tissue (BAT) as well as subcutaneous and epididymal WAT (sWAT and eWAT). The fractions of ILC2s, natural killer T (NKT) cells and eosinophils in sWAT, eWAT and BAT are significantly decreased by high-fat-diet (HFD) feeding and leptin deficiency-induced obesity. Consistent with this, the adipose expression and circulating levels of IL-33, a key inducing cytokine of ILC2, are significantly downregulated by obesity. Furthermore, administration of IL-33 markedly increases the fraction of ILC2 and eosinophil as well as the expression of UCP1 and tyrosine hydroxylase (TH), a rate-limiting enzyme in catecholamine biosynthesis, in adipose tissue of HFD-fed mice. On the other hand, cold exposure induces the expression levels of IL-33 and UCP1 and the population of ILC2 and eosinophil in sWAT, and these promoting effects of cold stress are reversed by neutralization of IL-33 signaling in vivo. Moreover, the basal and cold-induced IL-33 and ILC2/eosinophil pathways are significantly suppressed by sympathetic denervation via local injection of 6-hydroxydopamine (6-OHDA) in sWAT. Taken together, our data suggest that the ILC2/eosinophil axis in adipose tissue is regulated by sympathetic nervous system and obesity in IL-33-dependent manner, and IL-33-driven ILC2/eosinophil axis is implicated in the development of obesity.
\end{abstract} Key Words

- ILC2

- eosinophil

- sympathetic tone

- obesity

IL-33
Journal of Endocrinology (2016) 231, 35-48

\section{Introduction}

Beige or brite adipocytes, which are enriched in subcutaneous fat, have high thermogenic capacity and have become a new therapeutic target for the treatment of obesity and its related diseases (Akbari et al. 2003, Petrovic et al. 2010, Bostrom et al. 2012, Fisher et al. 2012). Type 2 immune responses are defined by the recruitment of 
$\mathrm{CD}^{+} \mathrm{T}$ helper type 2 (Th2) cells and the production of type 2 cytokines such as interleukin (IL)-4, IL-5, IL-9 and IL-13. An adipose tissue-specific type 2 immune response is characterized by infiltration of eosinophils, group 2 innate lymphoid cells (ILC2s), IL4- and/or IL13-induced M2 macrophages; production of type 2 cytokines; and plays a critical role in regulating browning of white adipose tissue (WAT), energy expenditure and glucose homeostasis (Molofsky et al. 2013, Brestoff et al. 2014, Khalifeh-Soltani et al. 2014, Rao et al. 2014). Type 2 cytokines including IL4, IL5 and IL13 are the key players in type 2 immunity and mediate its specific role in WAT browning. Deficiency of IL-4 and IL-13 impairs cold-induced WAT browning and exacerbates diet-induced obesity and insulin resistance (Rao et al. 2014). Macrophages are alternatively activated by IL-4 and IL-13 (IL-4/13) and mediate IL-4/13-induced browning by producing norepinephrine in adipose tissue (Wu et al. 2011, Van Dyken \& Locksley 2013). However, the production and regulation of type 2 cytokines in adipose tissue remains largely unknown.

Innate lymphoid cells are present in adipose tissue and promote type 2 inflammation and browning of fat (Brestoff et al. 2014, Lee et al. 2015). ILC2s, which were first discovered in the lung (Price et al. 2010), are activated by epithelial cell-derived cytokines IL-33 and IL-25 as well as thymic stromal lympoiphoidin in response to allergens. ILC2s orchestrate type 2 innate and adaptive immune responses (Koyasu \& Moro 2013, Licona-Limon et al. 2013, Cayrol \& Girard 2014). Very recently, ILC2s were identified in murine and human adipose tissue as a conserved characteristic of obesity, and have been shown to drive WAT browning and prevent the development of obesity (Brestoff et al. 2014, Lee et al. 2015). On one hand, ILC2s produce type 2 cytokines IL- 5 and IL-13, where IL-5 stimulates the maturation and infiltration of eosinophils and IL-13 activates M2 macrophages: eosinophils and M2 macrophages subsequently promote the browning effect in adipose tissue (Molofsky et al. 2013, Nussbaum et al. 2013, Khalifeh-Soltani et al. 2014, Lee et al. 2015). On the other hand, IL-33-elicited ILC2s themselves produce methionine-enkephalin peptides, which directly target adipocytes and promote browning and thermogenesis (Brestoff et al. 2014). In addition, natural killer T (NKT) cells found in human adipose tissue have been shown to produce and secrete IL-4, IL-5 and IL-13 (Akbari et al. 2003, Lynch et al. 2009, 2012). Invariant NKT cells are decreased in human obesity, and confer protection against the development of the metabolic syndrome and inflammation induced by high-fat-diet (HFD) feeding through anti-inflammatory cytokines such as IL-4 and IL-10 (Lynch et al. 2009, 2012). Adipose-resident eosinophils also produce type 2 cytokines, including IL-4, IL-5 and IL-13, and sustain adipose alternatively activated macrophages, thereby enhancing glucose tolerance (Wu et al. 2011). In contrast, $\gamma \delta$ T cells, another type of lymphocytic cells present in adipose tissue, are associated with diet-induced obesity, and have been shown to promote diet-induced inflammation and insulin resistance (Mehta et al. 2015). Although some studies suggest that lymphocytes in adipose tissue are involved in the development of obesity-induced inflammation and insulin resistance (Chatzigeorgiou et al. 2012, Mehta et al. 2015), whether various lymphocyte or lymphocytelike subsets in brown and beige adipose tissue play different roles in thermogenesis and obesity remains to be established. In addition, the $\beta 3$-adrenoceptor and its downstream protein kinase A (PKA) signaling pathway have been shown to drive the 'browning' effect in WAT in rodents (Cao et al. 2004). However, whether the $\beta 3$-adrenoceptor and its downstream signaling pathway play a role in regulating the recruitment and activation of ILC2s, NKT and $\gamma \delta$ T cells remains unclear.

Here, we show that the frequency of ILC2s, eosinophils and NKT cells in the stromal vascular fraction (SVF) is significantly lower in brown adipose tissue (BAT) and subcutaneous WAT (sWAT) compared with epididymal WAT (eWAT). Although the cell number of total lymphocytes and macrophages was increased by HFD feeding and leptin deficiency, the fractions of ILC2s, NKT cells and eosinophils in adipose tissue were significantly decreased by 12 weeks of HFD feeding and leptin deficiency. In support of this, the circulating and adipose expression levels of ILC2-inducing factor IL-33 were significantly downregulated by HFD feeding and leptin deficiency-induced obesity. Moreover, IL-33 administration dramatically upregulated the frequency of ILC2s and eosinophils and expression levels of UCP1 and tyrosine hydroxylase (TH) in sWAT of HFD-fed mice. Consistently, blocking IL-33 signaling with ST2 (IL-33 receptor) antibody suppressed the promoting effects of cold on ILC2/eosinophil axis and expression of UCP1 and $\mathrm{TH}$ in vivo. In addition, our data also suggest that sympathetic tone is required for cold-induced IL-33 production and recruitment of ILC2s and eosinophils in sWAT. Our data strongly suggest that the IL-33-driven ILC2/eosinophil axis in adipose tissue is a key pathway in the onset of obesity and may play an important role in regulating energy homeostasis. 


\section{Methods}

Mice Lepob (commonly referred to as $o b / o b$ ) heterozygous mice were purchased from Jackson Laboratory and intercrossed to generate $o b / o b$ homozygous mice and wild-type littermates. The lean wild-type mice were used as the control for $o b / o b$ homozygous mice. The $o b / o b$ heterozygous mice were only used for the generation of experimental mice but not for the following experiments. Six-week-old male C57BL/6 mice were fed either a normal chow diet (NCD) or HFD (45\% kcal from fat, D12451; Research Diets Inc, New Brunswick, NJ, USA) for $1,12,20$ or 32 weeks. All the animals were housed in the specific pathogen-free barrier facility with a $12 \mathrm{~h}$ light: $12 \mathrm{~h}$ darkness cycle with free access to food and water. All animal experimental protocols were reviewed and approved by the Animal Care Committee of the University of New Mexico Health Sciences Center.

\section{Denervation of inguinal fat pads}

Seven-week-old male C57BL/6 mice were anesthetized using constant flow isoflurane inhalation and a small incision was made in the abdominal skin. One of the two inguinal fat (sWAT) pads was denervated and the other pad, used as the control, was injected with the vehicle. 6-hydroxydopamine (6-OHDA, Sigma) was dissolved in PBS containing $1 \%$ ascorbic acid $(9 \mathrm{mg} / \mathrm{mL})$, and injected into the denervated pad using a $25 \mu \mathrm{L}$ Hamilton syringe $(2 \mu \mathrm{L}$ for each injection, total $24 \mu \mathrm{L} / \mathrm{pad}$ ) within $10 \mathrm{~min}$. The same volume of vehicle, PBS containing $1 \%$ ascorbic acid, was injected into the control fat pad. The needle was kept in place for $10 \mathrm{~s}$ after each injection to minimize reflux from the site of injection. The skin incision was closed with stainless steel wound clips and covered with nfz Puffer to protect the wound from infection after injection. The mice were then administered with $1 \mathrm{mg} / \mathrm{kg}$ of buprenorphine by intraperitoneal (i.p.) injection. Two weeks post denervation, half of operated mice were individually housed in cages with free access to food and water continuously and exposed to cold $\left(6^{\circ} \mathrm{C}\right)$ for 2 days and all were then killed. Inguinal fat was isolated for flow cytometry analysis.

Isolation of SVF The inguinal, epididymal and interscapular brown fat pads were collected to exclude lymph nodes, and $0.4 \mathrm{~g}$ inguinal fat, $0.5 \mathrm{~g}$ epididymal fat and $0.2 \mathrm{~g}$ brown fat were weighed and used for the isolation of SVF. The adipose tissues from different mice were individually digested with collagenase A (Roche), and the samples were filtered through $100 \mu \mathrm{M}$ cell strainers (Falcon) and washed with RPMI-1640 medium. The cell pellet was collected as SVF by centrifugation and resuspended for FACS analysis.

Flow cytometry The suspended SVF from adipose depots was fixed, blocked and stained with conjugated antibodies including anti-CD45, anti-Siglec-5, anti-CD11b and anti-CD206 (eBioscience and BioLegend) to identify macrophage subsets. To detect ILC2, the SVF cells were stimulated with a PMA/ionomycin/ monensin mixture and then incubated with antibodies to PE-conjugated anti-CD45, PerCP-conjugated anti-LIN (CD3e, CD11b, B220, CD11c, Gr-1, IgE) and Alexa 488-conjugated anti-IL-13 (eBioscience and BioLegend) after fixation and permeabilization. The $\mathrm{T}$ cell subpopulations were blocked and labeled with antibodies to PerCP-conjugated anti-CD3, APC-conjugated anti- $\gamma \delta$ TCR (eBioscience and BioLegend) and PE-conjugated anti-mCD1d/PBS-57 tetramer (NIH tetramer core facility) followed by fixation. FACS analysis was performed on a FACS Calibur (BD Pharmingen, San Diego, CA, USA), and the data were analyzed with FlowJo software as described previously (Yang et al. 2013).

\section{ELISA analysis of norepinephrine and IL-33}

Enzyme-linked immunosorbent assay (ELISA) of tissue samples for norepinephrine was performed following the manufacturer's protocol using a noradrenaline research ELISA kit (LDN Labor Diagnostika Nord GmbH \& Co. KG, Nordhorn, Germany). In brief, fat tissue was weighed and homogenized with $0.01 \mathrm{M} \mathrm{HCl}$ in the presence of $1 \mathrm{mM}$ EDTA and $4 \mathrm{mM}$ sodium metabisulfite. The norepinephrine was extracted and acylated from the tissue homogenate followed by enzyme conversion, and then measured by ELISA. And the ELISA analysis of IL-33 in serum and inguinal fat was performed following the manufacturer's protocol using a mouse IL-33 DuoSet ELISA kit (R\&D Systems).

Real-time PCR analysis Mouse inguinal fat samples were homogenized and the total RNA was isolated with the RNeasy Lipid Tissue Mini Kit (Qiagen). RNA (1 $\mu \mathrm{g})$ was reverse-transcribed using cDNA kit (Qiagen). PCR amplification was detected using SYBR Green PCR master mixture on Roche 480 Real-time PCR system (Roche).

Published by Bioscientifica Ltd. 

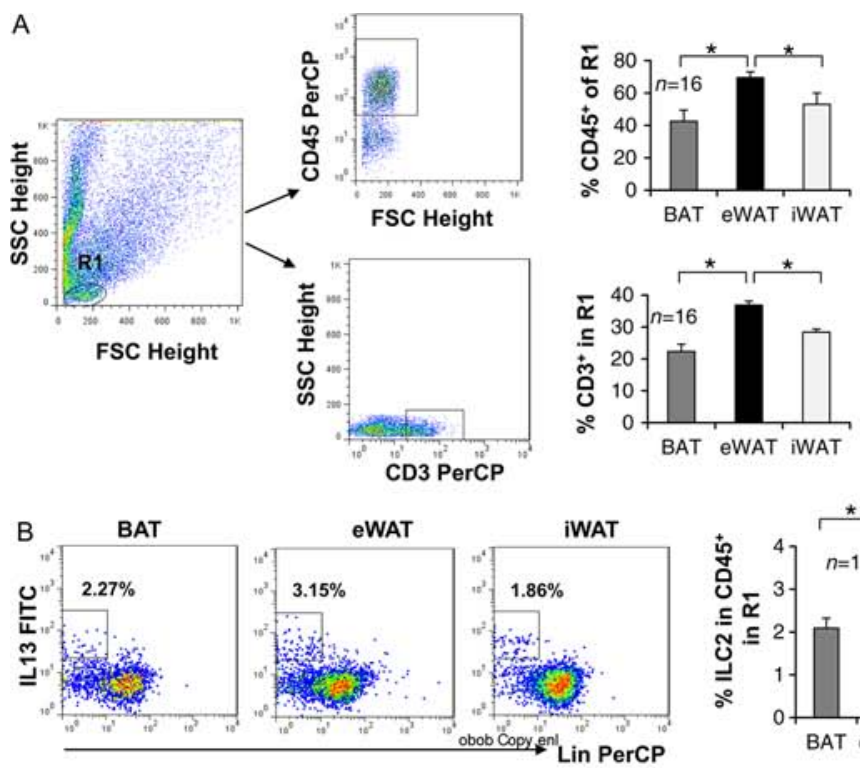

C
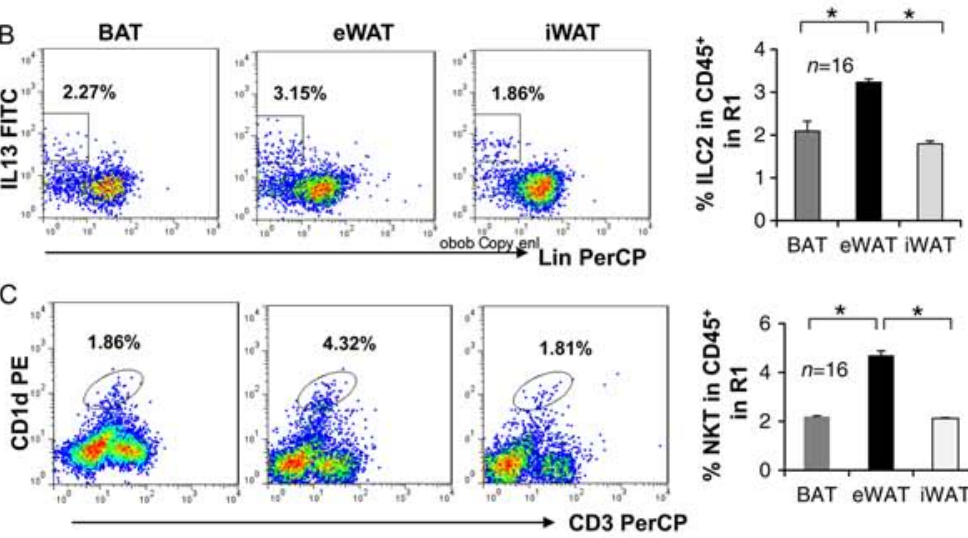

D
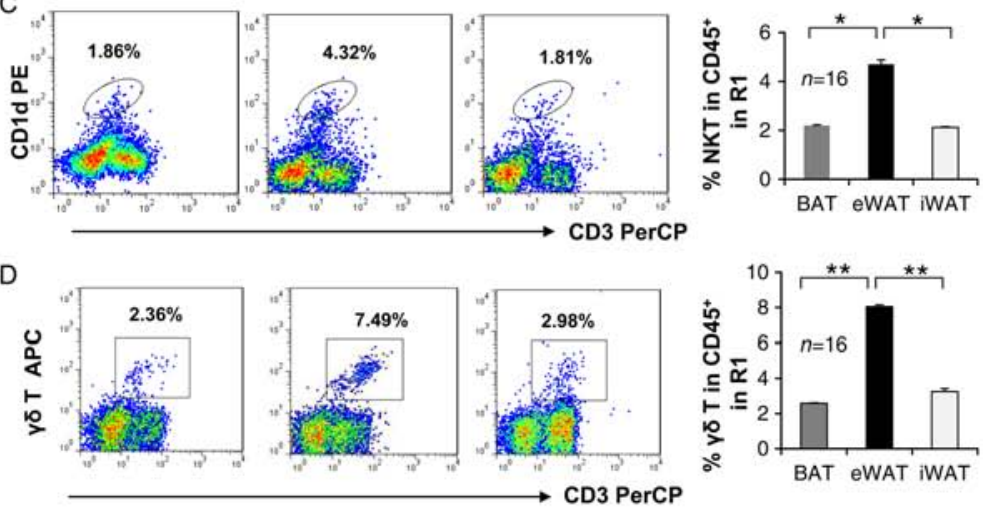

$\mathrm{E}$
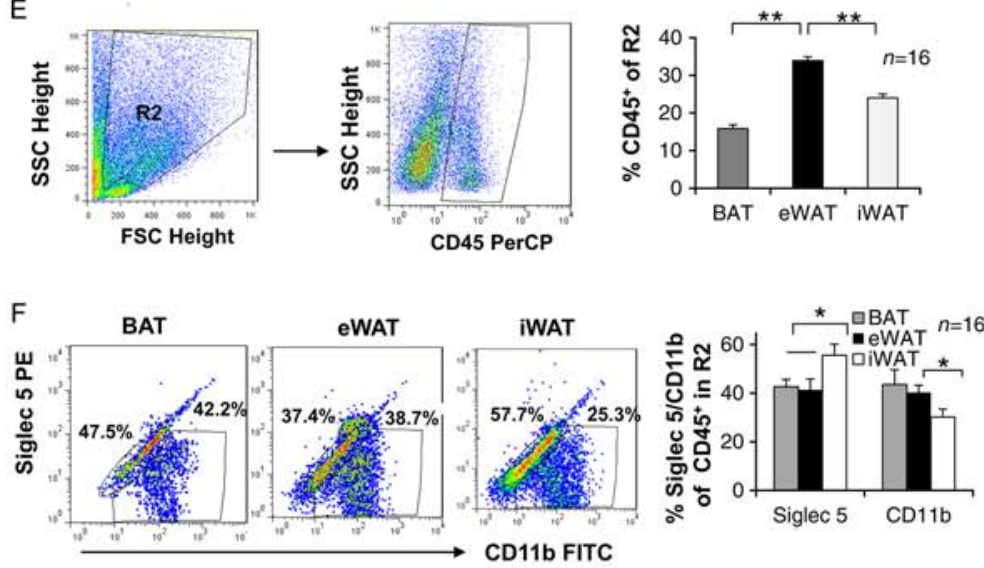

G
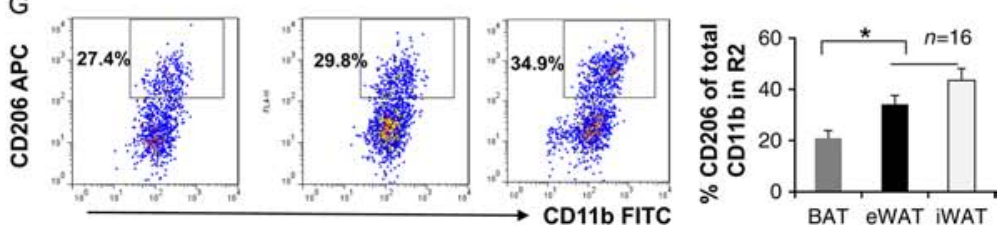

Figure 1

Differential abundance of innate/innate-like lymphocytes, eosinophils and monocytes in three types of fat. The SVF from 10-week-old male C57BL/6 mice $(n=16)$ was isolated, suspended, fixed, blocked, surface-stained, permeabilized and labeled by incubation with conjugate antibodies. The labeled cells were selected by size and granularity, and then gated by cell-surface markers using flow cytometry. (A) Definition of gate R1 (SSClowFSClow) cells, representative FACS plots and percentage of $\mathrm{FSClowCD} 45^{+}$and $\mathrm{SSClow} \mathrm{CD}_{3}{ }^{+}$ cells in gate $\mathrm{R} 1$ in brown, epididymal and inguinal fat pads. The dot plots depict SSC and FSC, and the sequential gating strategy for analysis of $\mathrm{CD}_{4} 5^{+}$and $\mathrm{CD}^{+}+$cells are presented. (B) Representative FACS plots of Lin-IL-13+ ILC2s in gate R1 and percentage of ILC2s in CD45+ cells from gate R1 in three fat pads. (C) Representative FACS plots of CD1d+CD3+ NKT cells in gate R1 and percentage of NKT cells in CD45+ cells from gate R1 in three fat pads. (D) Representative FACS plots of $\gamma \delta \mathrm{T}^{+} \mathrm{CD} 3^{+}$cells in gate $\mathrm{R} 1$ and percentage of $\gamma \delta$ T cells in CD45+ cells from gate R1 in three fat pads. (E) Definition of gate R2 (SSChighFSChigh) cells, representative FACS plots and percentage of CD45+ cells in gate R2 in three fat pads. (F) Representative dot plots and percentage of Siglec- $5+C D 11 b^{+}$and Siglec$5-C D 11 b+$ macrophages in CD45+ cells from gate R2. (G) Representative dot plots and percentage of Siglec$5-C D 11 b+C D 206+$ to Siglec 5-CD11b+ macrophages from gate R2 in three fat pads. The data were presented as the mean \pm s.E.M. $* P<0.05, * * P<0.01$. A full colour version of this figure is available at http://dx.doi.org/10.1530/ JOE-16-0229. http://joe.endocrinology-journals.org DOI: 10.1530/JOE-16-0229
๑) 2016 Society for Endocrinology Printed in Great Britain
Published by Bioscientifica Ltd 
The primer sequences were as follows: Arginase 1 forward primer 5'-CAGCTACCTGCTGGGAAGGAAGAA-3' and reverse primer 5'-CCAAGAGTTGGGTTCACTTCCA-3'; CD206 forward primer 5'-TGTGGTGAGCTGAAAGG TGA-3' and reverse primer 5'-CAGGTGTGGGCGCAG GTAG-3'; F4/80 forward primer 5'-CTTTGGCTATGGG CTTCCAGTC-3' and reverse primer 5'-GCAAGGAGGAC AGAGTTTATCGTG-3'; Nr3c2 forward primer 5'-GAAGAG CCCCTCTGTTTGCAG-3' and reverse primer 5'-TCCTT GAGTGATGGGACTGTG-3'; IL33 forward primer 5'-TGAG ACTCCGTTCTGGCCTC-3' and reverse primer 5'-CTCTT CATGCTTGGTACCCGAT-3'; Gapdh forward primer 5'-CGTGCCGCCTGGAGAAACC-3' and reverse primer 5'-TGGAAGAGTGGGAGTTGCTGTTG-3'. Each primer pair amplifies products spanning several exons, distinguishing spliced mRNA from genomic DNA contamination. The relative expression of target genes was normalized to GAPDH.

\section{Administration of IL-33}

Six-week-old male C57/BL6 mice were fed with HFD described above for 11 weeks and then administered with recombinant murine IL-33 (carrier-free, BioLegend) at $12.5 \mu \mathrm{g} / \mathrm{kg}$ body weight by i.p. injection for 1 week. The mice will be killed, and adipose tissue will be collected for flow cytometry and Western blot analyses.

\section{Administration of IL-33 receptor antibody}

Seven-month-old male C57/BL6 mice were administered with or without mouse ST2/IL-1 R4 antibody (R\&D Systems) at $100 \mu \mathrm{g} / \mathrm{mice} /$ day by i.p. injection under cold exposure $\left(6^{\circ} \mathrm{C}\right)$ for 2 days. The mice were killed, and adipose tissues were collected for flow cytometry analysis and Western blotting.

\section{Western blot analysis}

Fat tissue was homogenized, and the protein was normalized and separated with SDS-PAGE gel followed by immunoblots as described previously (Liu et al. 2012, 2014). The rabbit polyclonal anti-UCP1 and mouse monoclonal anti-tubulin antibodies were from Abcam. The phospho-PKA substrate and TH antibodies were purchased from cell signaling. The secondary antibodies including anti-rabbit IgG $(\mathrm{H}+\mathrm{L})$ HRP conjugate and anti-mouse IgG $(\mathrm{H}+\mathrm{L})$ AP conjugate were from Promega.

\section{Data analysis}

Statistical analysis was performed using a two-tailed Student's $t$-test between two groups or one-way ANOVA among three different groups. All the results were presented as the mean \pm s.E.M., and $P$ value of $<0.05$ was considered to be statistically significant.

\section{Results}

The abundance of ILC2s, NKT cells and $\gamma \delta$ T cells is lower in BAT and sWAT compared with eWAT

To investigate if lymphocyte population in adipose tissue is correlated with thermogenesis, we performed flow cytometry analysis for SVF isolated from interscapular brown, epididymal and inguinal fat pads. The viable cells in SVF were selected based on size, and gate R1 (SSClow FSC low) was defined for analysis of lymphocytes (Fig. 1A). The fraction of $\mathrm{CD}_{4} 5^{+}$cells (nucleated cells of hematopoietic origin) accounted for 48,78 and $58 \%$ of cells in gate R1 for brown, epididymal and inguinal fat, respectively (Fig. 1A). Consistently, the fraction of $\mathrm{CD}^{+}$cells ( $\mathrm{T}$ lymphocytes) within gate R1 was also significantly lower in brown (25\%) and inguinal (28\%) fat compared with epididymal fat (37\%) (Fig. 1A), suggesting that lymphocytes and $\mathrm{T}$ lymphocytes are enriched in eWAT. Since innate or innate-like lymphocytes including ILC2s, NKT and $\gamma \delta$ $\mathrm{T}$ cells reside in adipose tissue and have been shown to regulate energy or glucose homeostasis in WAT (Lynch et al. 2009, 2012, Brestoff et al. 2014, Lee et al. 2015, Mehta et al. 2015), we further examined the abundance of these lymphocytes in brown fat. Lin-IL-13+(ILC2s), $\mathrm{CD} 1 \mathrm{~d}$ tetramer $(\mathrm{CD} 1 \mathrm{~d} \text { tet })^{+} \mathrm{CD}^{+}+(\mathrm{NKT})$ and $\gamma \delta \mathrm{TCR}+{ }^{+} \mathrm{CD} 3^{+}$ $(\gamma \delta \mathrm{T})$ cells accounted for 3, 5 and $8 \%$ of total CD45+ cells within gate R1 in epididymal fat (Fig. 1B, C and D), suggesting the presence of an innate immune system within adipose tissue. In addition, the fractions of ILC2s, NKT and $\gamma \delta$ T cells were significantly lower in brown and inguinal fat compared with epididymal fat (Fig. 1B, C and D). There was no significant difference in ILC2s, NKT and $\gamma \delta \mathrm{T}$ cell fractions between brown and inguinal fat (Fig. 1B, C and D).

Given that ILC2s and NKT cells as well as Th2 cells produce type 2 cytokines such as IL- 4 and IL-13, well-known factors of eosinophil and M2 macrophage activation and promoters of WAT browning (Lynch et al. 2009, 2012, Brestoff et al. 2014, Han et al. 2014, Lee et al. 2015), we further investigated whether the macrophage

Published by Bioscientifica Ltd 
subset differs between brown, epididymal and inguinal fat. The cells in gate R2 (granulocytes) were defined and used for flow cytometry analysis of macrophage and eosinophil subsets (Fig. 1E). CD45+ cells accounted for 16,34 and $24 \%$ of cells in gate R2 for brown, epididymal and inguinal fat, respectively (Fig. 1E). The Siglec-5+CD11b+ (eosinophils, 37\%) and Siglec-5-CD11b+ (macrophages, 39\%) cells consisted mostly of CD45+ cells within gate R2 of epididymal fat (Fig. 1F). The percentage of eosinophil, the downstream effectors of ILC2 and NKT, was significantly greater in inguinal fat compared with brown and epididymal fat. Although the fraction of Siglec-5-CD11b+ macrophages within gate R2 was decreased in inguinal fat compared with epididymal fat, the percentage of Siglec- $5^{-} \mathrm{CD} 11 \mathrm{~b}+\mathrm{CD} 206^{+}(\mathrm{M} 2)$ to Siglec$5-\mathrm{CD} 11 \mathrm{~b}^{+}$macrophages was greater in inguinal compared with brown fat and epididymal fat (Fig. 1F and G). Taken together, our results suggest that ILC2s, NKT cells and $\gamma \delta$ $\mathrm{T}$ cells are present in BAT and sWAT as well as eWAT, and may play an important role in regulating the biology and function of adipose tissue.

\section{The frequency of ILC2s and eosinophils is driven by sympathetic tone in beige fat}

Activation of ILC2/eosinophil/macrophage axis is induced by cold exposure and promotes browning effect in WAT (Nguyen et al. 2010, Lee et al. 2015). Given that sympathetic tone mediates the cold-induced browning effect through norepinephrine in WAT (Farmer 2008, Barbatelli et al. 2010), we then examined whether the presence of these types of immune cells is driven by sympathetic innervation. We performed the denervation of inguinal fat using a local injection of 6-OHDA in 7-week-old male C57BL/6 mice followed by 2-week recovery and subsequent cold exposure. Pretreatment with 6-OHDA led to a marked reduction in basal and coldinduced norepinephrine production (Fig. 2A) as well as a robust downregulation of UCP1 expression in inguinal fat in vivo (Fig. 2B). Moreover, sympathetic denervation significantly suppressed basal and cold-induced fractions of ILC2s and eosinophils, while denervation had no significant effect on NKT and $\gamma \delta$ T cell fractions (Fig. 2C). Consistent with this, inhibiting sympathetic tone significantly decreased basal and cold-induced IL-33 levels in inguinal fat (Fig. 2D). These data suggest that sympathetic tone plays a critical role in regulating IL-33 production and the ILC2/eosinophil axis in beige fat. Notably, sympathetic denervation increased the basal and cold-induced $\mathrm{CD} 11 \mathrm{~b}^{+}$macrophages in beige fat despite a little effect on the fraction of CD206+ M2 macrophages under cold stress condition (Fig. 2E), suggesting that CD206 ${ }^{+}$M2 macrophages are not sensitive to sympathetic tone, and other M2 macrophage markers in addition to CD206 should be considered in the future study. These results indicate that IL-33 production and ILC2/eosinophil axis activation in beige fat are driven by norepinephrine, whereas M2 macrophages activation is not significantly altered by denervation.

\section{The abundance of ILC2s, eosinophils and NKT cells in SVF of adipose tissue is decreased by HFD feeding in vivo}

Previous studies show that the expression levels of UCP1 were significantly downregulated in sWAT by chronic HFD feeding (Fromme \& Klingenspor 2011, Shen et al. 2014). In contrast to SWAT, BAT UCP1 had been shown to be induced by HFD feeding (Fromme \& Klingenspor 2011, Shen et al. 2014). To further investigate the effect of HFD on UCP1 expression in BAT and sWAT, we performed time course study with HFD feeding for 1, 12, 20 and 32 weeks. We found that 1-12 weeks of HFD feeding upregulated the expression of UCP1 in BAT (Fig. 3B). Although UCP1 expression was slightly downregulated by 20 -weeks HFD feeding, this downregulation was not significant until 32-weeks HFD feeding (Fig. 3B). Different from BAT, UCP1 expression in sWAT was not significantly affected in 1 week but significantly suppressed from 12 to 32 weeks, suggesting that UCP1 expression in adipose tissue is regulated by overnutrition in duration-dependent manner, and the response in WAT is distinct from BAT. (Fig. 3A and B). To investigate the importance of innate/innate-like lymphocytes in adipose tissue under HFD feeding conditions, we analyzed brown, epididymal and inguinal fat from mice fed with NCD or HFD for 12 weeks for the presence of ILC2s, NKT and $\gamma \delta$ T cells using flow cytometry. The body mass was increased by $28 \%$ under HFD feeding (NCD mice average body weight 28.1 and $35.9 \mathrm{~g}$ for the mice fed with HFD (data not shown)). The fraction of $\mathrm{CD}_{4} 5^{+}$cells in gate $\mathrm{R} 1$ was also significantly increased by HFD feeding in all three types of fat (Fig. 3C), suggesting that lymphocyte abundance is positively correlated with the development of obesity. Consistent with this, the fraction of $\mathrm{CD}^{+}$cells in gate R1 was also slightly increased by HFD feeding in all three types of fat, although these differences did not reach statistical significance (Fig. 3D). Importantly, the fractions of ILC2s and NKT cells, but not $\gamma \delta$ T cells, in $\mathrm{CD} 45^{+}$cells of gate R1 were markedly decreased in all

Published by Bioscientifica Ltd. 
A

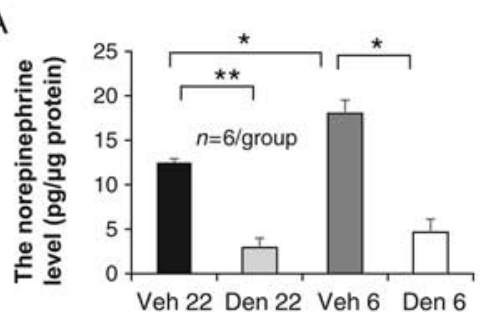

C

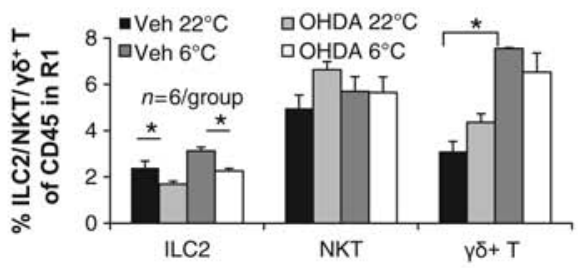

B

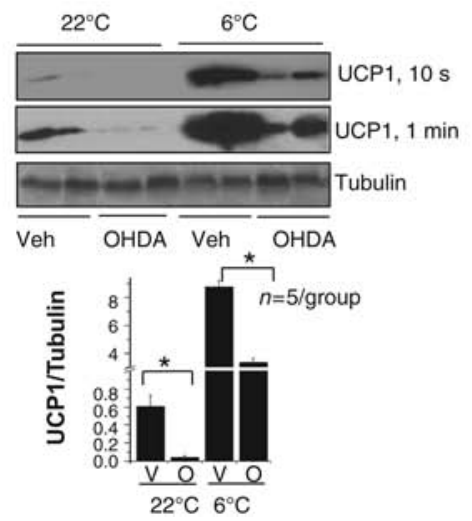

D

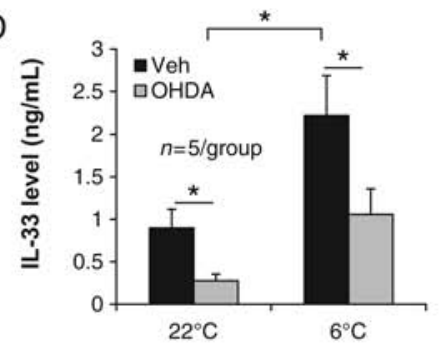

E

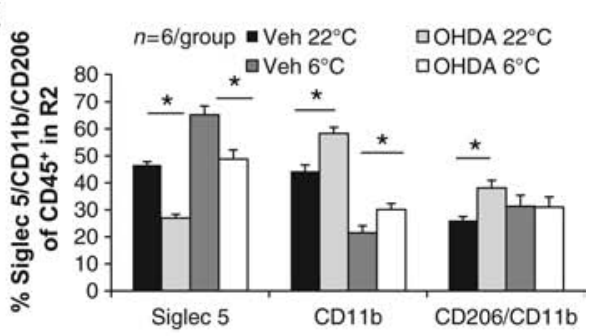

Figure 2

ILC2/eosinophil axis was suppressed by denervation in inguinal fat. Inguinal fat pad in 7-week-old male C57BL/6 mice ( $n=6)$ was locally injected with vehicle or 6-OHDA. Two weeks post injection, the mice were exposed with or without cold for $48 \mathrm{~h}$, and inguinal fat pads were collected for Western blot, ELISA and flow cytometry analysis. (A) The basal and cold-induced levels of norepinephrine in inguinal fat were significantly decreased by denervation. Inguinal fat pad was weighed, homogenized and used for the ELISA of norepinephrine. (B) The basal and cold-induced expression levels of UCP1 were greatly downregulated by denervation in inguinal fat. Tubulin was used as internal control. The data were quantified and analyzed with t-test. (C) The fractions of Lin-IL-13+ ILC2s but not CD1 + +CD $3+$ NKT cells and $\gamma \delta T$ cells under basal and cold conditions were decreased by denervation in inguinal fat. SVF from denervated or control inguinal fat was isolated and used for flow cytometry analysis. (D) The basal and cold-induced levels of IL-33 in inguinal fat were significantly decreased by denervation. Inguinal fat pads were homogenized and used for measurement of protein concentration and ELISA of IL-33. (E) Cold stress increased the percentage of Siglec-5+CD $11 \mathrm{~b}+$ eosinophils and decreased Siglec-5-CD $11 \mathrm{~b}+$ macrophage population, while denervation decreased the abundance of Siglec-5+CD11 b+ eosinophils and slightly increased Siglec $5-C D 11 b^{+}$macrophage population and percentage of Siglec $-5-C D 11 b+C D 206+(M 2)$ to Siglec $5-C D 11 b+$ macrophages. All data were presented as the mean \pm S.E.M. ${ }^{*} P<0.05, * \star P<0.01$.

three types of fat upon HFD feeding (Fig. 3E, F and G), suggesting that HFD feeding may selectively suppress the recruitment of ILC2s and NKT cells rather than $\gamma \delta$ $\mathrm{T}$ cells. In support of this, the percentage of eosinophils in $\mathrm{CD}_{45^{+}}$cells within gate $\mathrm{R} 2$ was also significantly decreased by HFD feeding despite an increase in total CD $45^{+}$cells in gate R2 in both brown and epididymal fat (Fig. 3H and I). These data point to a negative correlation between obesity and eosinophil abundance in adipose tissue. Notably, the infiltration of total macrophages into all three fat pads was markedly increased by HFD feeding (Fig. 3J), while the percentage of Siglec${ }^{-}-\mathrm{CD} 11 b^{+} \mathrm{CD} 206^{+}$(M2) to Siglec-5-CD11b+ macrophage was not significantly affected in any of three fat pads upon HFD feeding (Fig. 3K). Consistently, RT-PCR results show that the mRNA levels of macrophage marker F4/80, M1 marker Nr3c2 and M2 markers Arginase and CD206 are also induced by 12 weeks of HFD feeding (Fig. 3L), suggesting that macrophage differentiation and polarization may be associated with the stage of obesity development.

\section{ILC2 and NKT cell fractions in SVFs are decreased in adipose tissue of ob/ob mice}

To further evaluate the effect of obesity on the adipose innate immune system, we isolated adipose SVFs from 9-week-old male lean and $o b / o b$ mice and performed flow cytometry analysis. The body mass was increased by $38 \%$ in $o b / o b$ mice (31.4g) compared with lean mice (22.7 g) (data not shown). Intriguingly, expression levels of UCP1 were downregulated in inguinal fat and in BAT of $o b / o b$ mice compared with lean mice (Fig. 4A, B and C). Similar to HFD feeding, $\mathrm{CD}_{4} 5^{+}$but not $\mathrm{CD}^{+}$cell fractions in gate R1 was significantly increased in brown, epididymal and inguinal fat in $o b / o b$ mice compared with lean mice (Fig. 4D and E), indicating that lymphocyte infiltration into adipose tissue plays an important role 
A

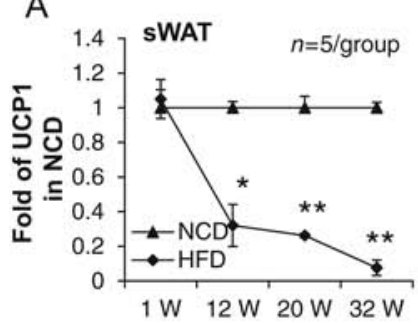

C
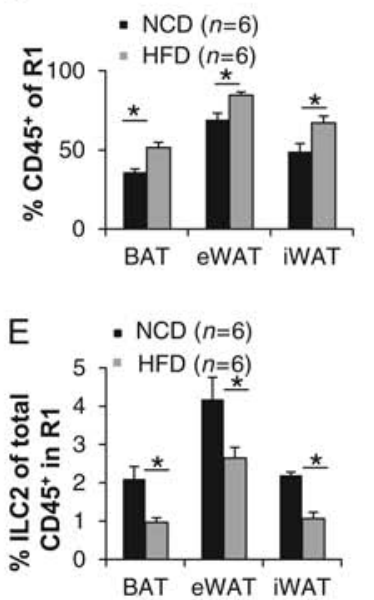

B

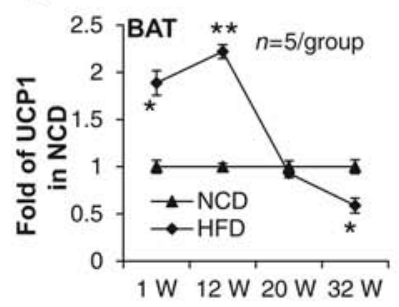

D

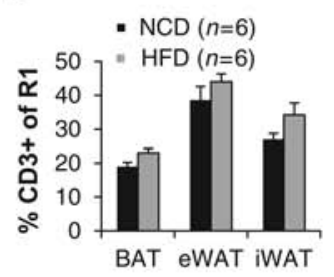

F

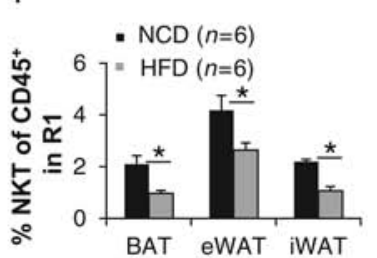

G

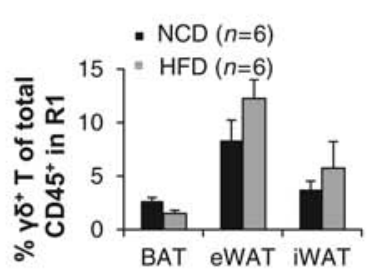

I
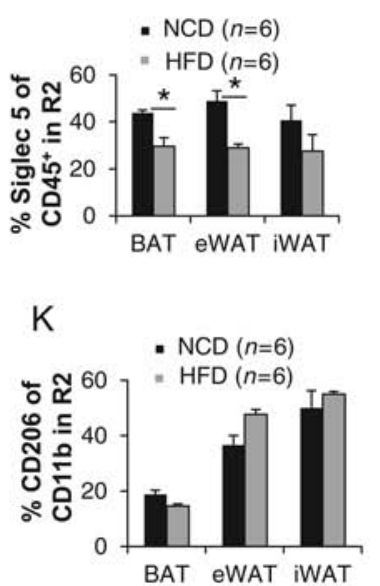

$\mathrm{H}$

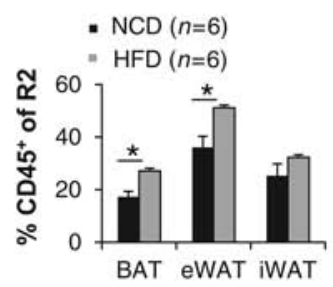

J
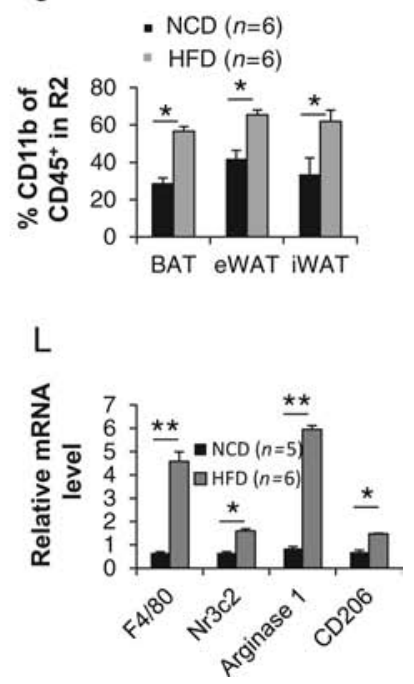

Figure 3

The abundance of ILC2s, eosinophils and NKT cells in SVF of adipose tissue was decreased by HFD feeding. Six-week-old C57BL/6 mice ( $n=6$ ) were fed with a normal chow diet (NCD) or high-fat diet (HFD) for 1, 12, 20 or 32 weeks. Western blot analysis was used to determine the expression levels of UCP1 in inguinal and brown fat in mice fed with NCD and HFD for 1, 12, 20 or 32 weeks. Tubulin was used as the loading control. The brown, inguinal and epididymal fat pads collected from mice fed with NCD or HFD for 12 weeks were used for flow cytometry analysis. The fold change in UCP1 expression of HFD feeding to NCD group in sWAT (A) and BAT (B) were quantified and analyzed. The percentage of FSClowCD45+ cells (C) but not SSClow $C D 3^{+}$cells (D) in gate R1 in three fat pads was significantly increased in HFD-fed mice compared with NCD-fed mice. The percentage of ILC2s (E) and NKT cells (F) but not $\gamma \delta \mathrm{T}(\mathrm{G})$ in CD45+ cells from gate R1 in three fat pads was significantly decreased by HFD feeding. (H) The percentage of FSClow CD45+ cells in gate R2 in three fat pads was significantly increased in HFD-fed mice compared with NCD-fed mice. (I) The fraction of Siglec $5+C D 11 b^{+}$eosinophils was decreased by HFD feeding. The population of Siglec $5-C D 11 b^{+}$macrophage $(J)$ but not Siglec-5-CD11 b+CD206+ (M2) to Siglec-5-CD11 b+ macrophage (K) was increased by HFD feeding. (L) The mRNA levels of macrophage marker F4/80, M1 marker Nr3c2 and M2 markers Arginase and CD206 are induced by 12 weeks of HFD feeding. The data were presented as the mean \pm S.E.M. ${ }^{*} P<0.05, * \star P<0.01$.

in the development of obesity. In addition, the fraction of ILC2s and NKT cells rather than $\gamma \delta$ T cells in gate R1 was significantly decreased in epididymal and inguinal fat pads of $o b / o b$ mice compared with lean mice (Fig. 4F, $\mathrm{G}$ and $\mathrm{H})$. ILC2 and NKT cell populations were slightly decreased in brown fat in $o b / o b$ mice, but the difference did not reach statistical significance (Fig. 4F, G and $\mathrm{H}$ ). Similar to HFD feeding conditions, where the CD45+ cell fraction in gate R2 was increased, leptin deficiency resulted in a significant decrease in the eosinophil fraction in all three fat pads (Fig. 4I and J). The infiltration of Siglec-5-CD $11 \mathrm{~b}^{+}$macrophages was increased (Fig. 4K), while the ratio of Siglec-5-CD $11 b^{+C D} 206^{+}$to Siglec$5^{-} \mathrm{CD} 11 b^{+}$macrophages was not significantly affected in any of the fat pads in $o b / o b$ mice compared with lean mice (Fig. 4L). Taken together, these data suggest a negative correlation between activation of ILC2/eosinophil axis, NKT cell infiltration and obesity.

\section{IL-33 administration improves ILC2/eosinophil pathway and the expression of UCP1 and TH in HFD-fed mice}

Next, we dissect the role of IL-33 in the suppression of ILC2/eosinophil axis and UCP1 by obesity given that IL-33 is a key inducting factor of ILC2s and WAT browning (Brestoff et al. 2014). We found that similar to UCP1 and ILC2/eosinophil pathway, mRNA and protein levels as well as circulating levels of IL-33 in inguinal WAT was induced by cold exposure and suppressed by HFD feeding and leptin deficiency-induced

Published by Bioscientifica Ltd 
A

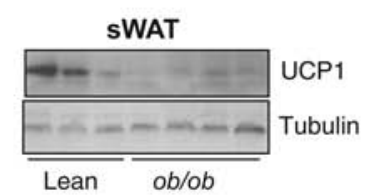

C
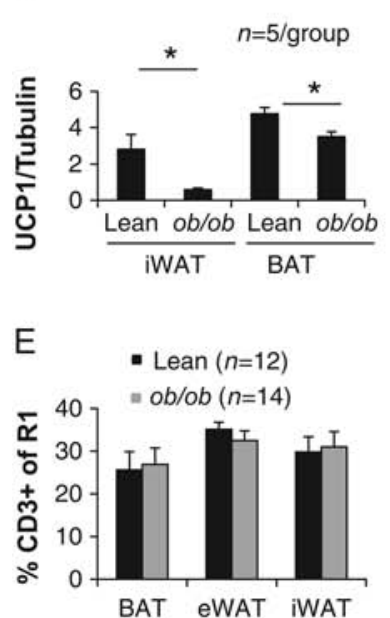

B

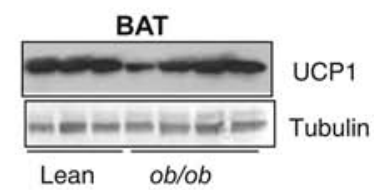

D

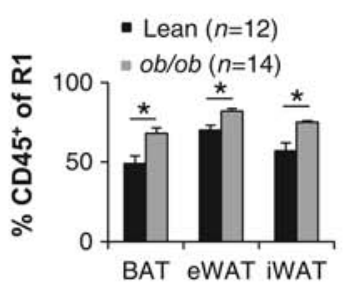

F

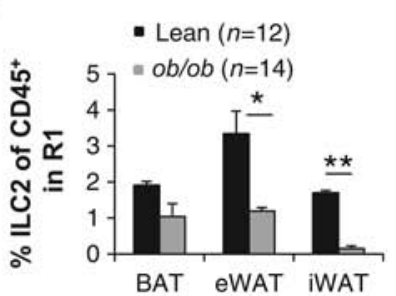

G

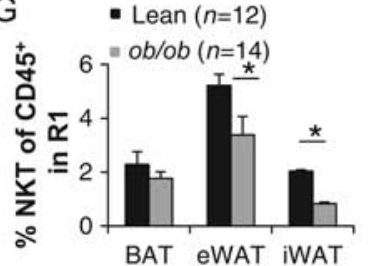

1

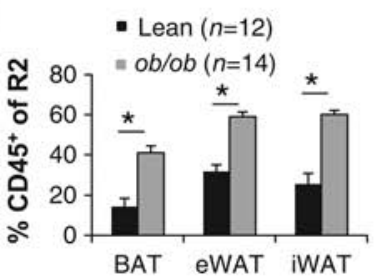

K

- Lean ( $n=12)$

- ob/ob (n=14)

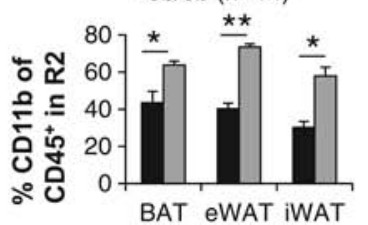

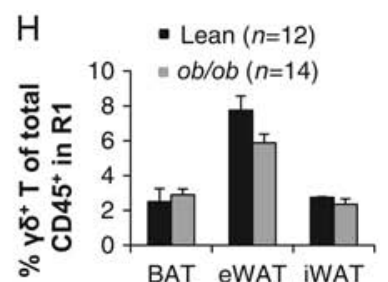

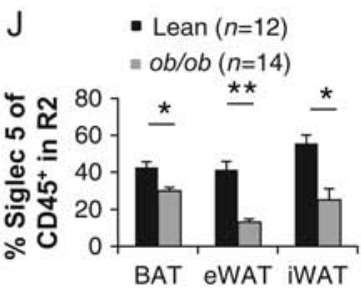

L

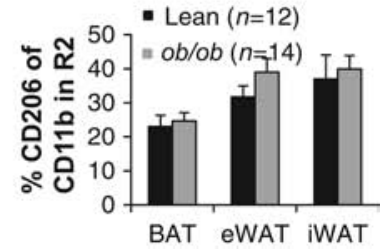

Figure 4

The fractions of NKT and ILC2s in SVF were decreased in brown, epididymal and inguinal fat in ob/ob mice. Brown, inguinal and epididymal fat pads were collected from 9-week-old lean $(n=12)$ and ob/ob $(n=14)$ mice, and SVF from fat was isolated and used for flow cytometry analysis. The expression levels of UCP1 in inguinal (A) and brown (B) fat were downregulated in ob/ob mice compared with lean mice. Tubulin was used as the loading control. (C) The data in Fig. 4A and B were quantified and analyzed. The percentage of FSClow CD45+ cells (D) but not SSClowCD3+ cells (E) in gate R1 in three fat pads was significantly increased in ob/ob mice compared with lean mice. The percentage of ILC2s (F) and NKT cells (G) but not $\gamma \delta$ T (H) in CD45+ cells from gate R1 in three fat pads was significantly decreased by leptin deficiency. (I) The percentage of SSChighFSChigh CD45+ cells in gate R2 in three fat pads was significantly increased in ob/ob mice compared with lean mice. (J) The fraction of Siglec $5+C D 11 b^{+}$eosinophils was decreased by leptin deficiency. The population of Siglec $5-C D 11 b+$ macrophage $(K)$ but not Siglec-5-CD11b+CD206+ (M2) to Siglec-5-CD11b+ macrophage (L) was increased by leptin deficiency. The data were presented as the mean \pm S.E.M. ${ }^{*} P<0.05, * * P<0.01$.

obesity (Fig. 5A, B and C), indicating that IL-33 downregulation may mediate the suppressing effect of obesity on ILC2/eosinophil axis and UCP1 in WAT. To address this question, 6-week-old male mice were fed with HFD for 11 weeks and then injected with vehicle or IL-33 for 7 days. The flow cytometry analysis showed that IL-33 administration notably increased the fraction of ILC2 in sWAT and eWAT about 13- and 9-folds, respectively, in HFD-fed mice (Fig. 5D and E). Consistent with this, IL-33 treatment significantly upregulated the expression levels of UCP1 in sWAT and eWAT (Fig. 5F). In agreement with this, IL-33 induced the expression of TH, a key enzyme of catecholamine biosynthesis and downstream of ILC2/eosinophil pathway in sWAT and eWAT of HFD-fed mice (Fig. 5G and H) (Tsao et al. 1998, Brestoff et al. 2014, Rao et al. 2014). Moreover, the phosphorylation of PKA substrates was enhanced by IL-33 administration in eWAT and sWAT (Fig. 5G and H).
These results suggest that IL-33 treatment greatly induces the WAT browning. However, IL-33 treatment had no significant effect on PKA substrate phosphorylation and UCP1 expression in BAT despite the increased ILC2 frequency (data not shown). Taken together, these results suggest that IL-33 plays a mediatory role in suppression of ILC2s/eosinophils and WAT browning by obesity, and downregulation of IL-33 in WAT is a potential marker for obesity.

\section{Cold-induced ILC2/eosinophil axis and expression of UCP1 and TH were suppressed by neutralization of IL-33 signaling}

To further determine the role of IL-33 in regulation of catecholamine production and thermogenesis, 7-month-old male mice were injected with vehicle or ST2 antibody under cold exposure condition for 2 days.

Published by Bioscientifica Ltd 
A

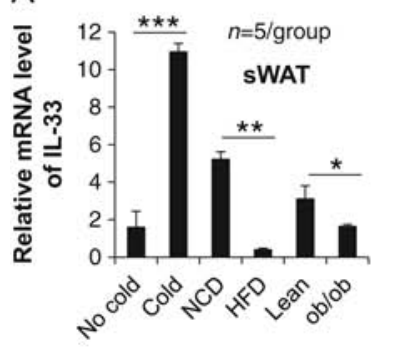

C

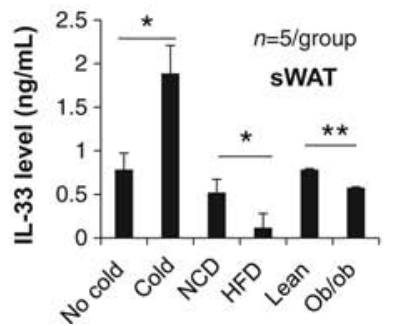

B

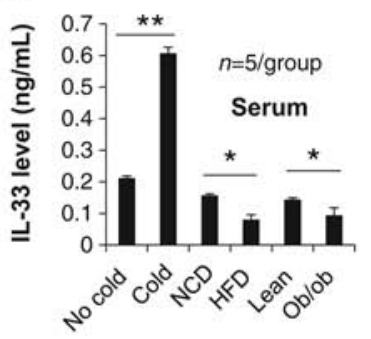

D

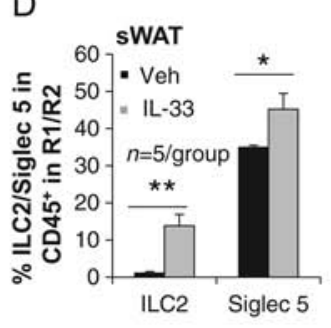

E
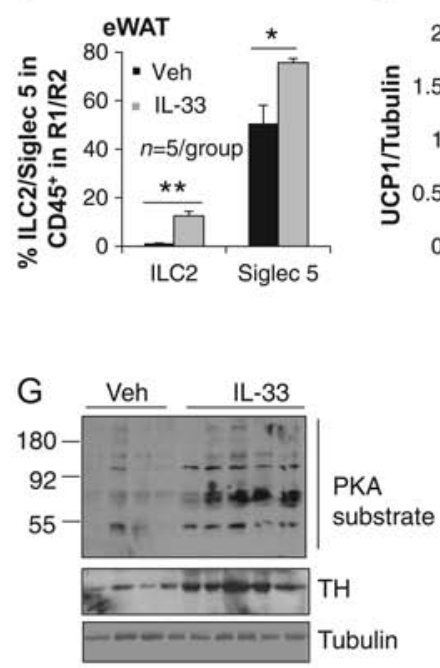

$\mathrm{F}$

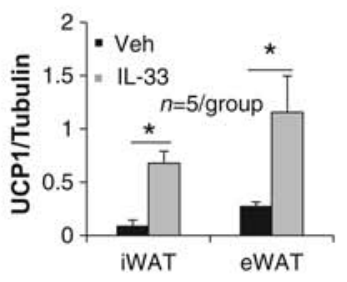

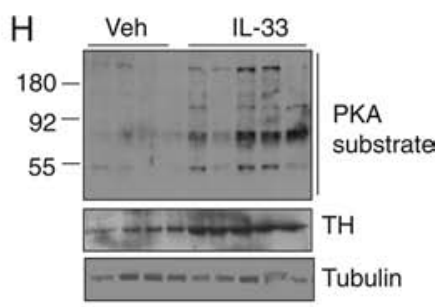

Figure 5

IL-33 treatment restored the fraction of ILC2/eosinophil pathway and UCP1 expression in WAT of HFD-fed mice. (A) The mRNA levels of IL-33 in inguinal fat were induced by cold exposure but suppressed by HFD feeding and leptin deficiency. The circulating levels (B) and protein level (C) in sWAT of IL-33 were significantly increased by cold stress, while decreased by HFD feeding and leptin deficiency. The level of IL-33 was measured with ELISA analysis. The percentages of ILC2s and eosinophils in CD45+ cells from gate R1 and gate R2, respectively, in sWAT (D) and eWAT (E) were significantly increased by IL-33 injection. (F) The protein levels of UCP1 in sWAT and eWAT were upregulated by IL-33 administration in HFD-fed mice. The expression levels of TH and phosphorylation of PKA substrates in sWAT (G) and eWAT (H) were upregulated by IL-33 administration in HFD-fed mice. Tubulin was used as the loading control. The data in Fig. 5A-F were presented as the mean \pm S.E.M. ${ }^{*} P<0.05, * * P<0.01, * * * P<0.001$.

Consistent with a previous finding of significant decrease in $\mathrm{CD}^{+} 0^{+} \mathrm{CD} 25^{+} \mathrm{ILCs}$ in both frequency and total cell number in the lungs by ST2 antibody administration (Monticelli et al. 2011), blocking IL-33 signaling decreased the fraction of ILC2 and eosinophil in sWAT and eWAT under cold stress condition (Fig. 6A and B). Moreover, neutralization of IL-33 signaling significantly downregulated the expression levels of UCP1 (Fig. 6C). In line with this, the expression levels of $\mathrm{TH}$ as well as PKA substrate phosphorylation were suppressed by the administration of ST2 antibody in sWAT and eWAT under cold stress condition (Fig. 6D and E). However, ST2 antibody administration had no inhibitory effect on PKA substrate phosphorylation and UCP1 expression despite a decrease in ILC2 fraction in BAT (data not shown). These results suggest that the induction of IL-33 is required for catecholamine production and WAT browning. However, the functional role of IL-33-driven ILC2 pathway in BAT is distinct from that in WAT.

\section{Discussion}

Type 2 inflammatory responses have been shown to play an important role in regulating browning of WAT through
M2 macrophage activation and type 2 cytokines release in eWAT and sWAT (Molofsky et al. 2013, Nussbaum et al. 2013, Brestoff et al. 2014, Khalifeh-Soltani et al. 2014, Rao et al. 2014). ILC2s reside in adipose tissue and drive the browning of WAT by producing type 2 cytokines including IL-5 and IL-13 in WAT (Lee et al. 2015). However, how the recruitment and activation of adipose-resident ILC2s are regulated remains unclear. In this study, we show that ILC2s are present in BAT and sWAT, and enriched in eWAT (Fig. 1). Moreover, the fraction of ILC2s is significantly decreased in BAT, sWAT and eWAT by HFD feeding and leptin deficiency (Figs 3 and 4). In addition, the abundance of ILC2/eosinophil axis as well as the levels of IL-33 and UCP1 are induced by cold exposure and suppressed by obesity in sWAT (Figs 2, 3 and 4). Given that ILC2s and eosinophils have been shown to promote the browning of WAT under cold stress (Brestoff et al. 2014, Khalifeh-Soltani et al. 2014, Rao et al. 2014, Lee et al. 2015), our data suggest that an ILC2/eosinophil axis exists in BAT as well as WAT and is regulated by both sympathetic tone and obesity.

IL-33 is a critical factor for the maintenance and stimulation of ILC2s in adipose tissue (Brestoff et al. 2014), and the circulating level of IL-33 has been shown to be downregulated by obesity, and IL-33 administration

Published by Bioscientifica Ltd 
A
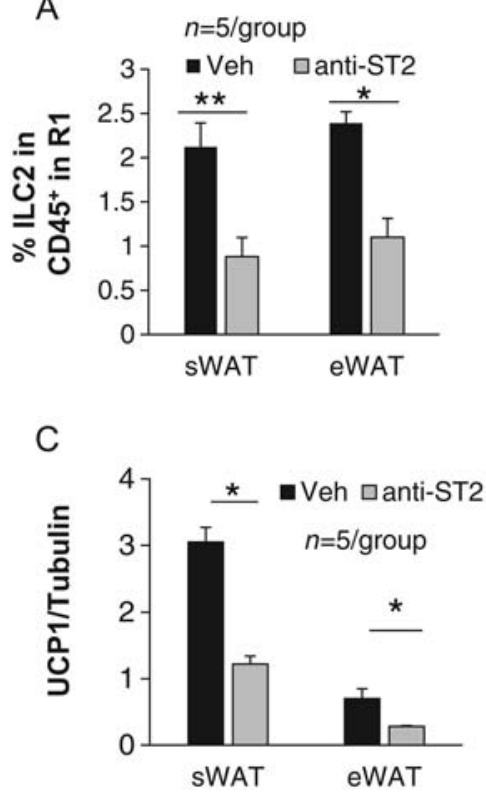

E

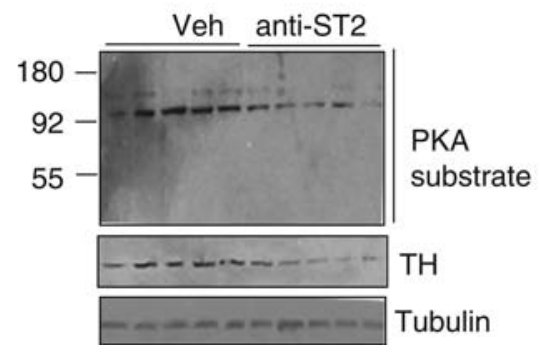

improves obesity and obesity-induced insulin resistance in vivo (Miller et al. 2010, Hasan et al. 2014, Han et al. 2015), implying that obesity may decrease ILC2 frequency in adipose tissue through downregulation of IL-33 production. Our study shows that IL-33 administration improves the percentage of ILC2s and eosinophils, catecholamine production and thermogenic gene expression in sWAT and eWAT of HFD-fed mice (Fig. 5). Our study suggests that ILC2-inducing cytokine IL-33 mediates the suppressing effect of obesity on ILC2/eosinophil axis and thermogenic gene expression in WAT, and is a potential marker of obesity. Consistent with this, neutralization of IL-33 signaling suppressed ILC2/eosinophil axis as well as catecholamine production and UCP1 expression in WAT under cold stress condition (Fig. 6). However, some beneficial effects of IL-33 are limited to WAT given that the promoting effects of IL-33 on catecholamine production and thermogenic gene expression are not observed in BAT despite the increased fraction of ILC2s in HFD-fed mice (Fig. 5 and data not shown). Our data are consistent with Brestoff's report that IL-33 treatment has no promoting effect on UCP1 expression, while increases ILC2 fraction in BAT (Brestoff et al. 2014). Although ILC2/eosinophil axis is suppressed by obesity in all three types of fat, IL-33/ILC2 pathway may have distinct effects in BAT compared with those in WAT. Our study also suggests that in addition to ILC2/ eosinophil axis, many other factors may be involved in the regulation of BAT activation. Along with this, adipose UCP1 expression is affected by HFD feeding in duration and tissue-dependent manners, and this response in WAT is distinguished from BAT (Fig. 3A and B).

Although IL-33 has been defined as an epithelial cellderived cytokine, recent studies also report that IL-33 as well as its receptor ST2, a protein that is encoded by the IL1RL1 gene in human adipose tissue, is predominantly detectable in endothelial cells (Price et al. 2010, Zeyda et al. 2013). It remains to be clarified if obesity suppresses IL-33 production through targeting of epithelial cells or endothelial cells. On the other hand, alternative macrophage activation is a downstream event of ILC2/eosinophil pathway. However, the ratio of Siglec$5^{-} \mathrm{CD} 11 b^{+}{ }^{-C D} 206^{+}$M2 macrophages to Siglec-5-CD11b ${ }^{+}$ macrophages is not significantly affected by HFD feeding 
and leptin deficiency (Figs 3 and 4). Moreover, the mRNA levels of both M1 and M2 markers are induced by HFD (Fig. 3L). Given that obesity leads to a shift in the activation state of adipose tissue macrophages from an M2-polarized state to an M1 proinflammatory state that contributes to insulin resistance (Lumeng et al. 2007), our data suggest that the effect of HFD feeding on differentiation and polarization of macrophage may be dynamic and needs to be further clarified. In addition, other markers of M2 macrophage such as galactose (Mgl-1) receptors, resistin-like molecule (RELM)- $\alpha$ and chitinases Ym1 and Ym2 should be included for the determination of M2 macrophage activation in future studies (Claria et al. 2011). Similar to HFD-fed mice, leptin deficiency leads to a decrease in ILC2/eosinophil axis activity and a downregulation of UCP1 expression in adipose tissue. Since leptin itself has been shown to positively regulate sympathetic tone and energy expenditure (Myers et al. 2009), the decreased browning and ILC2/eosinophil axis in fat depots of $o b / o b$ mice may be resulted from leptin deficiency itself as well as leptin deficiency-induced obesity as the secondary effect. In agreement with this, the ILC2 population is decreased in epididymal fat of obese rodents and human subjects (Brestoff et al. 2014).

The browning of WAT is controlled by sympathetic innervation in a $\beta 3$-adrenoceptor signaling pathwaydependent manner (Farmer 2008, Barbatelli et al. 2010). However, whether the abundance of ILC2s is regulated by norepinephrine remains unknown. Our study shows that inhibiting sympathetic tone by denervation impairs the abundance of ILC2s and expression of UCP1 in sWAT (Fig. 2), suggesting that $\beta 3$-adrenoceptor signaling pathway plays an important role in regulating ILC2s in adipose tissue. Consistently, inhibiting sympathetic tone significantly suppresses the level of IL-33 in sWAT (Fig. 2D). It has been shown that cytokines IL-33 and IL-25 as well as cold stress promotes the recruitment of ILC2s and browning effect in WAT (Hams et al. 2013, Brestoff et al. 2014, Lee et al. 2015). In addition, Lee and coworkers reported that environmental cold induces the recruitment of ILC2s via IL-33 (Lee et al. 2015). Our data suggest that activated sympathetic tone may promote ILC2 recruitment by upregulating the production of IL-33 in epithelial cells or other types of cells in response to cold or other environmental stimuli. In agreement with this, loss of IL-33 signaling downregulates ILC2/eosinophil pathway, norepinephrine biosynthesis and thermogenic gene expression in WAT under cold stress condition (Fig. 6). However, whether IL-33 in response to cold or overnutrition is locally produced in fat or enters through the circulation needs to be established in the future. While activation of the ILC2-eosinophil axis has been shown to drive M2 macrophage polarization and browning (Lee et al. 2015), our data show that sympathetic tone plays a critical role in cold-induced browning and ILC2-eosinophil axis activation, but not M2 macrophage polarization, suggesting that ILC2/eosinophil activation and M2 macrophage activation may be disassociated and mediated through two independent mechanisms under some particular conditions. Consistent with this, Brestoff and coworkers reported that ILC2s themselves promote the browning of fat through self-derived factor enkephalin, but do not confer browning through macrophage-dependent mechanisms (Brestoff et al. 2014).

In summary, our study identifies sympathetic tone as a key regulator of ILC2/eosinophil axis in beige fat. In addition, inactivation of IL-33/ILC2/eosinophil pathway is a novel hallmark of obesity, and this pathway may play an important role in regulating thermogenesis and obesity development.

\section{Declaration of interest}

The authors declare that there is no conflict of interest that could be perceived as prejudicing the impartiality of the research reported.

\section{Funding}

Junior Faculty Research Award (1-13-JF-37 to M L) from the American Diabetes Association; Grant-in-Aid Award (\#15GRNT24940018 to M L) from American Heart Association; CoBRE Pilot Award associated with P30 (P30GM103400 (PI: J Liu)) (to M L) and RAC Pilot Award (to M L) at the University of New Mexico Health Sciences Center (UNMHSC); American Lung Association RG-268131; and UNM Cancer Center Pilot Award FIG1186 (through contract P30CA118100) (to X Y).

\section{Acknowledgements}

The mCD1d/PBS-57 tetramer is provided by NIH tetramer core facility (contract HHSN272201300006C). We thank Jesse Denson at the UNMHSC for her editing this manuscript.

\section{References}

Akbari O, Stock P, Meyer E, Kronenberg M, Sidobre S, Nakayama T, Taniguchi M, Grusby MJ, DeKruyff RH \& Umetsu DT 2003 Essential role of NKT cells producing IL-4 and IL-13 in the development of allergen-induced airway hyperreactivity. Nature Medicine 9 582-588. (doi:10.1038/nm851)

Barbatelli G, Murano I, Madsen L, Hao Q, Jimenez M, Kristiansen K, Giacobino JP, De Matteis R \& Cinti S 2010 The emergence of cold-induced brown adipocytes in mouse white fat depots is determined predominantly by white to brown adipocyte transdifferentiation. American Journal of Physiology: Endocrinology and Metabolism 298 E1244-E1253. (doi:10.1152/ajpendo.00600.2009)

Published by Bioscientifica Ltd. 
Bostrom P, Wu J, Jedrychowski MP, Korde A, Ye L, Lo JC, Rasbach KA, Bostrom EA, Choi JH, Long JZ, et al. 2012 A PGC1-alpha-dependent myokine that drives brown-fat-like development of white fat and thermogenesis. Nature 481 463-468. (doi:10.1038/nature10777)

Brestoff JR, Kim BS, Saenz SA, Stine RR, Monticelli LA, Sonnenberg GF, Thome JJ, Farber DL, Lutfy K, Seale P, et al. 2014 Group 2 innate lymphoid cells promote beiging of white adipose tissue and limit obesity. Nature 519 242-246. (doi:10.1038/nature14115)

Cao W, Daniel KW, Robidoux J, Puigserver P, Medvedev AV, Bai X, Floering LM, Spiegelman BM \& Collins S 2004 p38 mitogen-activated protein kinase is the central regulator of cyclic AMP-dependent transcription of the brown fat uncoupling protein 1 gene. Molecular Cell Biology 24 3057-3067. (doi:10.1128/MCB.24.7.3057-3067.2004)

Cayrol C \& Girard JP 2014 IL-33: an alarmin cytokine with crucial roles in innate immunity, inflammation and allergy. Current Opinion in Immunology 31 31-37. (doi:10.1016/j.coi.2014.09.004)

Chatzigeorgiou A, Karalis KP, Bornstein SR \& Chavakis T 2012 Lymphocytes in obesity-related adipose tissue inflammation. Diabetologia 55 2583-2592. (doi:10.1007/s00125-012-2607-0)

Claria J, Gonzalez-Periz A, Lopez-Vicario C, Rius B \& Titos E 2011 New insights into the role of macrophages in adipose tissue inflammation and fatty liver disease: modulation by endogenous omega-3 fatty acid-derived lipid mediators. Frontiers in Immunology 249. (doi:10.3389/fimmu.2011.00049)

Farmer SR 2008 Molecular determinants of brown adipocyte formation and function. Genes \& Development 22 1269-1275. (doi:10.1101/ gad.1681308)

Fisher FM, Kleiner S, Douris N, Fox EC, Mepani RJ, Verdeguer F, Wu J, Kharitonenkov A, Flier JS, Maratos-Flier E, et al. 2012 FGF21 regulates PGC-1alpha and browning of white adipose tissues in adaptive thermogenesis. Genes \& Development 26 271-281. (doi:10.1101/ gad.177857.111)

Fromme T \& Klingenspor M 2011 Uncoupling protein 1 expression and high-fat diets. American Journal of Physiology: Regulatory, Integrative and Comparative Physiology 300 R1-R8. (doi:10.1152/ ajpregu.00411.2010)

Hams E, Locksley RM, McKenzie AN \& Fallon PG 2013 Cutting edge: IL-25 elicits innate lymphoid type 2 and type II NKT cells that regulate obesity in mice. Journal of Immunology 191 5349-5353. (doi:10.4049/jimmunol.1301176)

Han J, Lee E, Kim E, Yeom MH, Kwon O, Yoon TH, Lee TR \& Kim K 2014 Role of epidermal gammadelta T-cell-derived interleukin 13 in the skin-whitening effect of Ginsenoside F1. Experimental Dermatology 23 860-862. (doi:10.1111/exd.12531)

Han JM, Wu D, Denroche HC, Yao Y, Verchere CB \& Levings MK 2015 IL-33 Reverses an obesity-induced deficit in visceral adipose tissue ST2+ T regulatory cells and ameliorates adipose tissue inflammation and insulin resistance. Journal of Immunology 194 4777-4783. (doi:10.4049/jimmunol.1500020)

Hasan A, Al-Ghimlas F, Warsame S, Al-Hubail A, Ahmad R, Bennakhi A, Al-Arouj M, Behbehani K, Dehbi M \& Dermime S 2014 IL-33 is negatively associated with the BMI and confers a protective lipid/ metabolic profile in non-diabetic but not diabetic subjects. BMC Immunology 15 19. (doi:10.1186/1471-2172-15-19)

Khalifeh-Soltani A, McKleroy W, Sakuma S, Cheung YY, Tharp K, Qiu Y, Turner SM, Chawla A, Stahl A \& Atabai K 2014 Mfge8 promotes obesity by mediating the uptake of dietary fats and serum fatty acids. Nature Medicine 20 175-183. (doi:10.1038/nm.3450)

Koyasu S \& Moro K 2013 Th2-type innate immune responses mediated by natural helper cells. Annals of the New York Academy of Sciences 1283 43-49. (doi:10.1111/nyas.12106)

Lee MW, Odegaard JI, Mukundan L, Qiu Y, Molofsky AB, Nussbaum JC, Yun K, Locksley RM \& Chawla A 2015 Activated type 2 innate lymphoid cells regulate beige fat biogenesis. Cell 160 74-87. (doi:10.1016/j.cell.2014.12.011)
Licona-Limon P, Kim LK, Palm NW \& Flavell RA 2013 TH2, allergy and group 2 innate lymphoid cells. Nature Immunology 14 536-542. (doi:10.1038/ni.2617)

Liu M, Xiang R, Wilk SA, Zhang N, Sloane LB, Azarnoush K, Zhou L, Chen H, Xiang G, Walter CA, et al. 2012 Fat-specific DsbA-L overexpression promotes adiponectin multimerization and protects mice from diet-induced obesity and insulin resistance. Diabetes $\mathbf{6 1}$ 2776-2786. (doi:10.2337/db12-0169)

Liu M, Bai J, He S, Villarreal R, Hu D, Zhang C, Yang X, Liang H, Slaga TJ, Yu Y, et al. 2014 Grb10 promotes lipolysis and thermogenesis by phosphorylation-dependent feedback inhibition of mTORC1. Cell Metabolism 19 967-980. (doi:10.1016/j.cmet.2014.03.018)

Lumeng CN, Bodzin JL \& Saltiel AR 2007 Obesity induces a phenotypic switch in adipose tissue macrophage polarization. Journal of Clinical Investigation 117 175-184. (doi:10.1172/JCI29881)

Lynch L, O'Shea D, Winter DC, Geoghegan J, Doherty DG \& O'Farrelly C 2009 Invariant NKT cells and CD1d(+) cells amass in human omentum and are depleted in patients with cancer and obesity. European Journal of Immunology 39 1893-1901. (doi:10.1002/eji.200939349)

Lynch L, Nowak M, Varghese B, Clark J, Hogan AE, Toxavidis V, Balk SP, O'Shea D, O'Farrelly C \& Exley MA 2012 Adipose tissue invariant NKT cells protect against diet-induced obesity and metabolic disorder through regulatory cytokine production. Immunity 37 574-587. (doi:10.1016/j.immuni.2012.06.016)

Mehta P, Nuotio-Antar AM \& Smith CW 2015 gammadelta T cells promote inflammation and insulin resistance during high fat dietinduced obesity in mice. Journal of Leukocyte Biology 97 121-134. (doi:10.1189/jlb.3A0414-211RR)

Miller AM, Asquith DL, Hueber AJ, Anderson LA, Holmes WM, McKenzie AN, Xu D, Sattar N, McInnes IB \& Liew FY 2010 Interleukin-33 induces protective effects in adipose tissue inflammation during obesity in mice. Circulation Research $\mathbf{1 0 7}$ 650-658. (doi:10.1161/CIRCRESAHA.110.218867)

Molofsky AB, Nussbaum JC, Liang HE, Van Dyken SJ, Cheng LE, Mohapatra A, Chawla A \& Locksley RM 2013 Innate lymphoid type 2 cells sustain visceral adipose tissue eosinophils and alternatively activated macrophages. Journal of Experimental Medicine 210 535-549. (doi:10.1084/jem.20121964)

Monticelli LA, Sonnenberg GF, Abt MC, Alenghat T, Ziegler CG, Doering TA, Angelosanto JM, Laidlaw BJ, Yang CY, Sathaliyawala T, et al. 2011 Innate lymphoid cells promote lung-tissue homeostasis after infection with influenza virus. Nature Immunology 12 1045-1054. (doi:10.1038/ni.2131)

Myers MG Jr, Munzberg H, Leinninger GM \& Leshan RL 2009 The geometry of leptin action in the brain: more complicated than a simple ARC. Cell Metabolism 9 117-123. (doi:10.1016/j.cmet.2008.12.001)

Nguyen KD, Qiu Y, Cui X, Goh YP, Mwangi J, David T, Mukundan L, Brombacher F, Locksley RM \& Chawla A 2010 Alternatively activated macrophages produce catecholamines to sustain adaptive thermogenesis. Nature 480 104-108. (doi:10.1038/nature10653)

Nussbaum JC, Van Dyken SJ, von Moltke J, Cheng LE, Mohapatra A, Molofsky AB, Thornton EE, Krummel MF, Chawla A, Liang HE, et al. 2013 Type 2 innate lymphoid cells control eosinophil homeostasis. Nature 502 245-248. (doi:10.1038/nature12526)

Petrovic N, Walden TB, Shabalina IG, Timmons JA, Cannon B \& Nedergaard J 2010 Chronic peroxisome proliferator-activated receptor gamma (PPARgamma) activation of epididymally derived white adipocyte cultures reveals a population of thermogenically competent, UCP1-containing adipocytes molecularly distinct from classic brown adipocytes. Journal of Biological Chemistry $\mathbf{2 8 5}$ 7153-7164. (doi:10.1074/jbc.M109.053942)

Price AE, Liang HE, Sullivan BM, Reinhardt RL, Eisley CJ, Erle DJ \& Locksley RM 2010 Systemically dispersed innate IL-13-expressing cells in type 2 immunity. PNAS 107 11489-11494. (doi:10.1073/ pnas.1003988107) http://joe.endocrinology-journals.org

DOI: $10.1530 / J O E-16-0229$
(C) 2016 Society for Endocrinology Printed in Great Britain 
Rao RR, Long JZ, White JP, Svensson KJ, Lou J, Lokurkar I, Jedrychowski MP, Ruas JL, Wrann CD, Lo JC, et al. 2014 Meteorin-like is a hormone that regulates immune-adipose interactions to increase beige fat thermogenesis. Cell 157 1279-1291. (doi:10.1016/j.cell.2014.03.065)

Shen Y, Song SJ, Keum N \& Park T 2014 Olive leaf extract attenuates obesity in high-fat diet-fed mice by modulating the expression of molecules involved in adipogenesis and thermogenesis. EvidenceBased Complementary and Alternative Medicine 2014971890. (doi:10.1155/2014/971890)

Tsao CW, Lin YS \& Cheng JT 1998 Inhibition of immune cell proliferation with haloperidol and relationship of tyrosine hydroxylase expression to immune cell growth. Life Sciences $\mathbf{6 2}$ PL335-PL344. (doi:10.1016/s0024-3205(98)00170-2)

Van Dyken SJ \& Locksley RM 2013 Interleukin-4- and interleukin-13mediated alternatively activated macrophages: roles in homeostasis and disease. Annual Review of Immunology 31 317-343. (doi:10.1146/ annurev-immunol-032712-095906)

Wu D, Molofsky AB, Liang HE, Ricardo-Gonzalez RR, Jouihan HA, Bando JK, Chawla A \& Locksley RM 2011 Eosinophils sustain adipose alternatively activated macrophages associated with glucose homeostasis. Science 332 243-247. (doi:10.1126/science.1201475)

Yang XO, Zhang H, Kim BS, Niu X, Peng J, Chen Y, Kerketta R, Lee YH, Chang SH, Corry DB, et al. 2013 The signaling suppressor CIS controls proallergic T cell development and allergic airway inflammation. Nature Immunology 14 732-740. (doi:10.1038/ni.2633)

Zeyda M, Wernly B, Demyanets S, Kaun C, Hammerle M, Hantusch B, Schranz M, Neuhofer A, Itariu BK, Keck M, et al. 2013 Severe obesity increases adipose tissue expression of interleukin-33 and its receptor ST2, both predominantly detectable in endothelial cells of human adipose tissue. International Journal of Obesity 37 658-665. (doi:10.1038/ijo.2012.118)

Received in final form 25 July 2016 Accepted 1 August 2016
C 2016 Society for Endocrinology Printed in Great Britain
Published by Bioscientifica Ltd. 